2017-11-15

Numerical models for the motion and forces of point-absorbing wave energy converters in extreme waves

Sjokvist, L

http://hdl.handle.net/10026.1/10366

10.1016/j.oceaneng.2017.08.061

Ocean Engineering

Elsevier BV

All content in PEARL is protected by copyright law. Author manuscripts are made available in accordance with publisher policies. Please cite only the published version using the details provided on the item record or document. In the absence of an open licence (e.g. Creative Commons), permissions for further reuse of content should be sought from the publisher or author. 


\title{
Numerical models for the motion and forces of point-absorbing wave energy converters in extreme waves
}

\author{
Linnea Sjökvist ${ }^{\mathrm{a}, \mathrm{b}, \mathrm{e}, *}$, Jinming $\mathrm{Wu}^{\mathrm{c}, \mathrm{e}}$, Edward Ransley ${ }^{\mathrm{d}}$, Jens Engström ${ }^{\mathrm{a}}$, \\ Mikael Eriksson ${ }^{\mathrm{a}}$, Malin Göteman ${ }^{\mathrm{a}}$ \\ ${ }^{a}$ Department of Engineering Sciences, Uppsala University, Uppsala, Sweden \\ ${ }^{b}$ Center for Natural Disaster Science (CNDS), Villavägen 16, SE-752 36 Uppsala, Sweden \\ ${ }^{c}$ Department of Mechanical Engineering, Harbin Institute of Technology, Harbin, China \\ ${ }^{d}$ School of Marine Science and Engineering, Plymouth University, UK \\ ${ }^{e}$ Authors contributed to the manuscript equally
}

\begin{abstract}
Reliable simulation tools are necessary to study performance and survivability of wave energy devices, since experiments are both expensive and difficult to implement. In particular, survivability in nonlinear, high waves is one of the largest challenges for wave energy, and since the wave loads and dynamics are largely model dependent, each device must be studied separately with validated tools. In this paper, two numerical methods based on fully nonlinear computational fluid dynamics (CFD) are presented and compared with a simpler linear method. All three methods are compared and validated against experimental data for a point-absorbing wave energy converter in nonlinear, high waves. The wave energy converter consists of a floating buoy attached to a linear generator situated on the seabed. The line forces and motion of the buoy are studied, and computational cost and accuracy are compared and discussed. Whereas the simpler linear method is very fast, its accuracy is not sufficient in high and extreme waves, where instead the computationally costly CFD methods are required. The two CFD models are considered validated with the physical wave

\footnotetext{
* Corresponding author

Email addresses: linnea.sjokvist@angstrom.uu.se (Linnea Sjökvist), wujinming@hit.edu.cn (Jinming Wu), edward.ransley@plymouth.ac.uk (Edward Ransley), jens.engstrom@angstrom.uu.se (Jens Engström), mikael.eriksson@angstrom.uu.se (Mikael Eriksson), malin.goteman@angstrom.uu.se (Malin Göteman)
}

Preprint submitted to Journal of Ocean Engineering 
tank experiment. The OpenFOAM model showed a higher accuracy, but also a higher computational cost than the ANSYS Fluent model.

Keywords: Wave power, CFD, peak force, extreme wave, physical experiment

\section{Introduction}

\subsection{Background}

The realization of full-scale wave energy systems requires fast and reliable simulation tools that can study the performance of the system with many de-

5 grees of freedom and for a large range of parameters. A wave energy converter (WEC) system is most thoroughly described by solving the Navier-Stokes and power take off (PTO) equations (often non-linear) simultaneously. This approach is very computationally time consuming, and even though it may be necessary for extreme design cases, it is not a suitable approach for optimization design studies. A wide range of simplifications and restrictions are possible, from assuming a linear PTO to using linear potential flow theory for the simulated waves. Extensive analytical work has been done on optimizing energy absorption by point-absorbing floating bodies restrained by linear PTO systems and for monochrome waves during the 1970s 1, 2, 3, 4, 5. If a linear PTO and regular waves with small amplitude are assumed, the hydrodynamic forces on the floating body can be decomposed into hydrodynamical parameters, and numerical modeling can be used to simulate the WECs behavior in the frequency domain [6, 7. Time-domain modeling based on the hydrodynamical parameters was developed in the 1980's [8]. This linearisation is widely used, and has been proven to show acceptable agreement for low and moderate sea states [9, 10]. However, in order for wave energy to be a viable energy option, the survivability in harsh offshore environments must also be guaranteed, which includes surviving forces in extreme wave events. The magnitude of these forces and the dynamic behaviour of the WEC must be found, so that the WEC can be properly designed. 
For high sea states and extreme waves, the flow behaviour around a WEC will be turbulent, overturning and often highly nonlinear and can be approximated with e.g. the Reynolds Average Navier-Stokes (RANS) equations together with a turbulence model [11. Numerical models based on the finite element method 30 (FEM) or the finite volume method (FVM) can then be used to solve the RANS equations, and the interface between two phases can be calculated using the volume of fluid method (VOF). RANS-VOF is an accurate nonlinear model [12], and can be used both to identify hydromechanical parameters or full state dynamics of floating bodies [13, 14, or to model a complete WEC system during an extreme wave event. Several CFD models of WECs have been experimentally verified; in reference [15] the motion of a flap type WEC modelled in OpenFOAM shows good agreement with experiment; in [16], a 2-body point-absorber is modelled in heave motion; in [17] and [18] a point-absorbing WEC with linearelastic mooring, moving in six degrees of freedom, is modelled showing good 40 agreement with wave tank experiments, and; in reference [19], another pointabsorber was modelled both fixed and freely floating.

For the WEC concept developed at Uppsala University, Sweden, the line force has been measured offshore in both full scale during normal operating conditions [20, 21] and in a scaled model test with linear springs instead of 45 a generator in larger seas [22]. However, the offshore environment does not provide the controlled environment needed to make a qualitative analysis. In a 1:20 scale experiment performed in a physical wavetank, the endstop force was analysed and seen to decrease with an increased applied frictional damping, also called Coulomb damping, 23. The survivability of the Uppsala University 50 WEC is numerically studied in [24, using a RANS-VOF model verified with experimental data reprinted from [23. The PTO damping in the verification experiment was nonlinear but low. However, as one possiblility for limiting damaging peak forces is to increase the PTO damping [23], the model response to PTO damping is an important issue that is addressed in this paper. 


\subsection{Paper objective}

Although numerical methods have been used to study offshore structures in energetic sea states before, the dynamics and forces involved remain highly dependent on the structure being modelled. In contrast to traditional offshore structures, it is also of importance to correctly account for the PTO damping in

60 a WEC, as it is strongly coupled to the WECs behaviour [25]. Only a few studies have been published on point-absorbing WECs with a linear generator and limited stroke length [24]. The survivability can be studied experimentally, but experiments in a wave tank can be difficult and expensive, and reliable computational methods are needed. However, they need to be verified with physical data

65 and compared with each other to find the most reliable and efficient methods for solving the underlying equations.

This paper presents three numerical models studying the motion, the line force and the peak forces of a WEC system, and compares them with each other and with experimental wave tank data. Two of the models, an OpenFOAM model and an ANSYS Fluent model, are RANS-VOF solvers. The third model is a linear potential flow model, using coupled equations of motion in MATLAB, with hydromechanical parameters computed using WAMIT. The PTO damping is modelled as Coulomb damping (dry friction), and each model's performance is studied with different damping conditions. The elasticity of the connection line in the physical experiment is accounted for when the endstop spring is hit.

The WEC system in this paper is comprised of a freely-floating surface piercing buoy, coupled to a translator on the seabed, which is constrained to move vertically. This model corresponds to the full-scale wave energy devices that have been developed and constructed by Uppsala University since 2002. A 80 schematic figure and a photograph of the WEC can be seen in figure 1. 


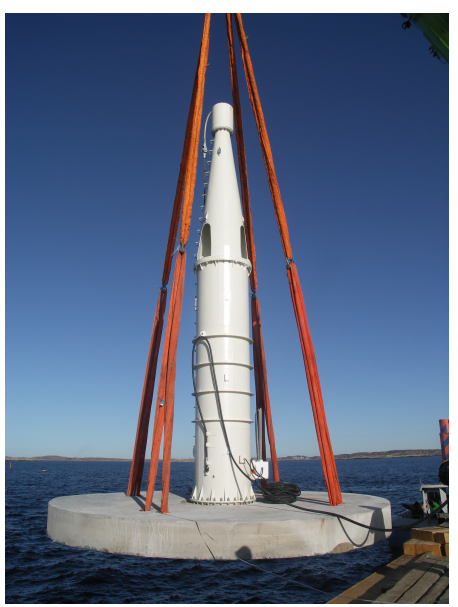

(a) A linear generator before deployment

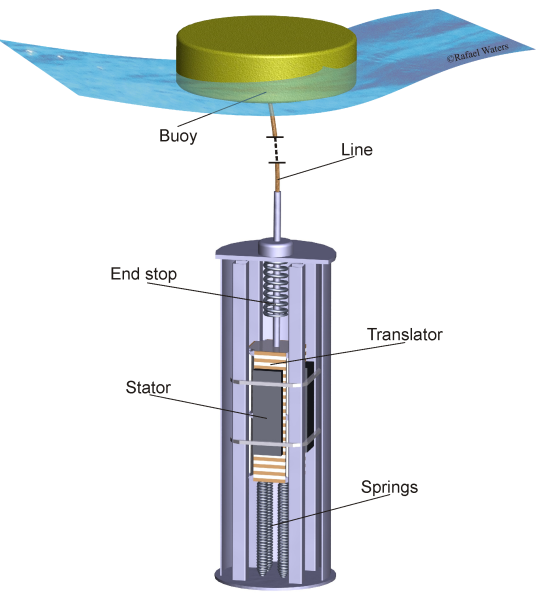

(b) The main parts of the WEC

Figure 1: a) A full scale WEC generator is photographed just before offshore deployment. This particular prototype is known as the L9 generator and is here mounted on a concrete gravity based foundation. The buoy was connected the day after the generator deployment. b) The translator is directly driven by the floating buoy, generating electricity as it moves inside the stator.

\section{Methodology}

\subsection{WEC description}

The system under investigation here is the point-absorbing WEC developed at Uppsala University. The WEC consists of a direct-drive linear generator situated on the seabed connected to a surface buoy by a connection line, (figure 1). The chosen parameters, found in table 1, are appropriate parameters for WECs at the offshore test site outside Lysekil, Sweden [26]. For engineering usability, the numerical models are run at full-scale, as presented in the table, where as the physical experiments were run at 1:20 scale. In the physical experiments, the PTO-damping, which depends on the translator velocity, was modelled using a friction damper. For comparability, it is implemented as Coulomb damping (dry friction) in all the numerical models in this paper.

Two buoy geometries have been modelled: a cylinder (CYL) and a cylinder 
with a moonpool (CWM). The geometry of the two buoys are shown in figure 2 . water damping in overtopping waves.

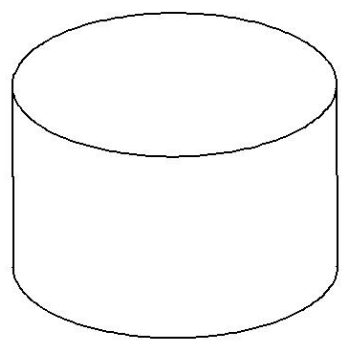

(a) Cylindrical buoy (CYL).

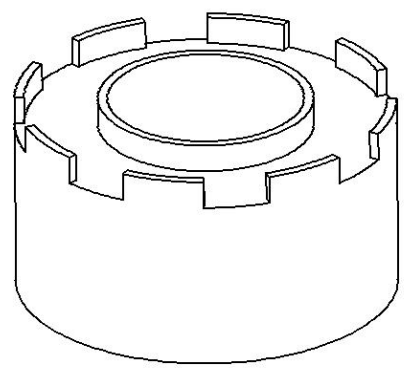

(b) Cylindrical buoy with moonpool (CWM), and water damping top.

Figure 2: The geometry of the modelled buoys. The height is $2.12 \mathrm{~m}$ for both buoys. For the CYL buoy, the radius is $1.7 \mathrm{~m}$, and for the CWM buoy, the outer and inner radius are $2 \mathrm{~m}$ and $1.03 \mathrm{~m}$ respectively.

\subsection{Equations of motion of the WEC system}

The floating buoy is subject to three translational degrees of freedom and three rotational, whereas the translator is constrained to vertical motion only. The two bodies are connected by a connection line, see figure 3 .

This two-body system has two motion states; either they move together or they move separately, according to whether or not the connecting line is tensioned. The position of the buoy, relative to its equilibrium position, is denoted as $\bar{r}_{b}(t)=\left(x_{b}(t), y_{b}(t), z_{b}(t)\right)$. The position of the translator is defined by a single vertical coordinate $z_{t}(t)$, with $z_{t}=0$ denoting the equilibrium position. The equilibrium is defined as where the buoy is at rest in still water. The connection points between the connection line and buoy or translator are denoted $O_{b}$ and $O_{t}$ respectively, see figure 3

The hydrodynamic forces $\bar{F}_{p}$ and torque $\bar{M}_{p}$ acting on the rigid body are obtained by integrating the fluid pressure over the surface area of the buoy. The 


\begin{tabular}{|lll|}
\hline Parameters & Symbol & Values \\
\hline Buoy outer radius CYL / CWM & $R$ & $1.7 \mathrm{~m} / 2 \mathrm{~m}$ \\
Buoy inner radius CWM & $R_{\text {in }}$ & $1.03 \mathrm{~m}$ \\
Buoy height CYL / CWM & $h$ & $2.12 \mathrm{~m} / 2.12 \mathrm{~m}$ \\
Buoy draft CYL / CWM & $d$ & $1.3 \mathrm{~m} \mathrm{/} \mathrm{1.6} \mathrm{m}$ \\
Buoy mass CYL / CWM & $m_{b}$ & $5736 \mathrm{~kg} / 8592 \mathrm{~kg}$ \\
Translator mass & $m_{t}$ & $6240 \mathrm{~kg}$ \\
Upper endstop spring & $\kappa$ & $776 \mathrm{kN} / \mathrm{m}$ \\
Upper endstop spring length & $l_{\kappa}$ & $0.6 \mathrm{~m}$ \\
Maximal stroke length upper / lower & $\xi_{u p} / \xi_{\text {down }}$ & $1.8 \mathrm{~m} / 1.8 \mathrm{~m}$ \\
Spring constant of connection line & $\Lambda$ & $300 \mathrm{kN} / \mathrm{m}$ \\
Generator damping & $\mu$ & $0,18,59,83 \mathrm{kN}$ \\
Water depth & $D$ & $50 \mathrm{~m}$ \\
\hline
\end{tabular}

Table 1: WEC and system specifications.

line force, $\bar{F}_{\text {line }}$, acting on the buoy is directed along a unit vector between $O_{b}$ and $O_{t}$. The total force acting on the body is then the hydrodynamic forces, $\bar{F}_{p}$, plus the line force, $\bar{F}_{\text {line }}$, and the gravitational force. The equations of motion for the buoy and the translator are connected by the line force, as long as the connection line is tensioned,

$$
\begin{aligned}
m_{b} \ddot{\ddot{r}} & =\int p \hat{n} d S+F_{\text {line }} \hat{r}-m_{b} g \hat{z}, \\
J_{b} \ddot{\ddot{\theta}} & =\int \bar{r}_{0} \times p \hat{n} d S+\bar{r}_{c} \times F_{\text {line }} \hat{r}, \\
m_{t} \ddot{z}_{t} & =F_{\text {line }}-m_{t} g+F_{\mathrm{PTO}}+F_{\kappa}+F_{\text {stop }},
\end{aligned}
$$

where the first two equations describe the three translational and rotational degrees of freedom of the buoy respectively and the third equation describes the vertical motion of the translator. In the equations, $p$ is the pressure on the buoy surface, $\hat{n}$ is the unit vector normal to the buoy surface, $g$ is the acceleration due to gravity, $\hat{z}$ is the unit vector in the $\mathrm{z}$ direction, $J_{b}$ is the principle moment of inertia of the buoy, $\theta$ is the angular motion of the buoy, $\bar{r}_{0}$ is the vector from 
$O_{b}$ to the centre of the buoy, and $\bar{r}_{c}$ is the vector from $O_{t}$ to the buoy centre. Since the buoy and the translator are related by the line force, the forces on the translator will act as restraining forces on the buoy. In the physical validation experiments the electromagnetic damping of the full scale generator, $F_{\mathrm{PTO}}$, is modelled using frictional damping. To be comparable, the generator damping in the simulations is also modelled as a constant force, $\mu$, directed in the opposite direction of the translator motion. A ramping function is used so that

$$
F_{\mathrm{PTO}}= \begin{cases}-\mu, & \delta<\dot{z}_{t}(t), \\ -\mu \frac{\dot{z}_{t}}{\delta}, & -\delta \leq \dot{z}_{t}(t) \leq \delta, \\ \mu, & \dot{z}_{t}(t)<-\delta,\end{cases}
$$

where $\delta$ is a translator velocity of $0.2 \mathrm{~m} / \mathrm{s}$. By adding a ramping function, the simulations gain stability, which was needed in the ANSYS model. The OpenFOAM model was run both with and without the ramping function, assuring that neither the buoy motion or the peak forces were affected by the ramping function. It did however provide a smoother simulated line force for velocities close to zero, which better resembles the experimental situation. When the translator hits the upper endstop spring, the endstop will add an additional restraining force $F_{\kappa}$, which is given as

$$
F_{\kappa}= \begin{cases}-\kappa z_{t}(t), & z_{t}(t)>\xi_{\text {up }}-l_{\kappa}, \\ 0, & \text { otherwise }\end{cases}
$$

where $\kappa$ is the spring constant of the end-stop spring. The translator has a limited stroke length $\xi_{\text {down }} \leq z_{t}(t) \leq \xi_{\text {up }}$. When this is exceeded the connection line will act as an elastic mooring line, adding a further restraining force, $F_{\text {stop }}$. However, in the physical experiment, it was seen that the spring constant corresponding to the elasticity of the connection line was lower than the spring constant of the end-stop spring. To handle this, $F_{\text {stop }}$ was set to zero and when the end-stop spring was fully compressed, $\kappa$ was reset to the spring constant of the connection line. Unless the endstop-spring was fully compressed, $\kappa$ was recalculated as the corresponding spring constant of a compressing spring (the endstop-spring) and an elongated spring (the connection 
line). When $z_{t}(t)<\xi_{\text {down }}$, the translator rests on the lower endstop and the line is slack, the line force is then set to zero.

The forces due to the endstop spring and the elasticity of the line are discontinuous and only act on the translator when it reaches the limited stroke lengths, which is modelled using the Heaviside function. In the case when the connecting line is slack, the line force is set to zero, and the movement of the two bodies become uncoupled and have to be considered separately. The switch point for above two cases depends on the line force and the distance between the buoy and the translator. When the line force becomes negative, the connecting line starts to slack. When the distance between the buoy and the translator exceeds the non-tensioned length of the line, the connecting line starts to be tensioned.

\subsection{Two-phase Navier-Stokes models}

Two different CFD models are used in this paper, an OpenFOAM model and an ANSYS model. Both solve the RANS equation using the FVM, and in both cases the water surface is tracked using the VOF method. The equations for the air and the water are written assuming a single fluid mixture governed by the equations:

$$
\begin{gathered}
\nabla \cdot \bar{u}=0 \\
\frac{\partial}{\partial t}(\rho \bar{u})+\nabla \cdot\left(\rho\left(\bar{u}-\bar{u}_{g}\right) \bar{u}\right)=-\nabla p+\nabla \cdot \bar{S}+\rho \bar{f}_{b}
\end{gathered}
$$

where $\bar{u}$ is the fluid velocity and $\bar{u}_{g}$ is the grid velocity, $\rho$ is the mixture density and $p$ is the pressure. The viscous stress tensor is $\bar{S}=2 \mu \bar{D}$, where $\mu$ is the mixture viscosity and $\bar{D}$ is the rate of strain tensor. The force from a rigid body is $f_{b}$. The VOF method uses a scalar field for the phase fraction, $\alpha$, to track the two fluids, where $\alpha=1$ denotes water and $\alpha=0$ denotes air, and values in between denote a mixture of the two phases. An additional transport equation is used to solve $\alpha$ :

$$
\frac{\partial \alpha}{\partial t}+\nabla \cdot\left(\alpha\left(\bar{u}-\bar{u}_{g}\right)\right)=0
$$




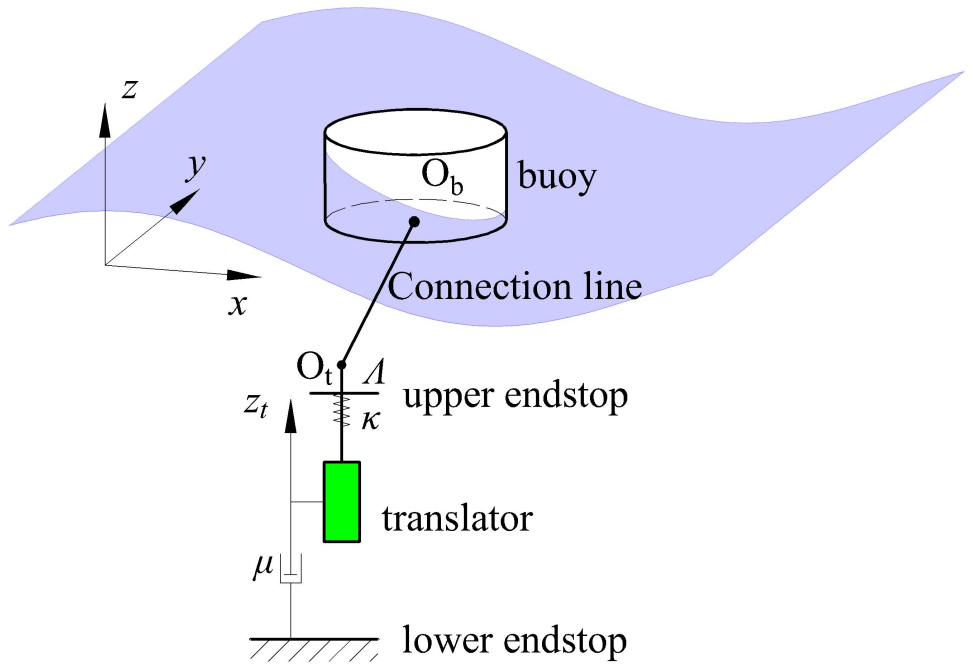

Figure 3: Schematic figure of the system. The buoy and the translator are connected by a connection line. Whereas the translator is restricted to move only vertically, the buoy is free to move in several degrees of freedom.

The fluid properties in the mixture, $\Phi$, can then be expressed in terms of $\alpha$ :

$$
\Phi=\alpha \Phi_{\text {water }}+(1-\alpha) \Phi_{\text {air }}
$$

where $\Phi$ is a fluid property such as $\mu$ or $\rho$. The turbulence, in both CFD models, is simulated with the RNG $\mathrm{k}-\epsilon$ turbulence model.

\subsubsection{The OpenFOAM model}

The OpenFOAM model is solved using OpenFOAM v.2.4.0 and the twophase Navier-Stokes solver interDyMFoam. A 3D moving mesh is used and the dynamic mesh is handled by the sixDoFRigidBodyMotion solver. The computational domain is $300 \mathrm{~m} \times 60 \mathrm{~m} \times 100 \mathrm{~m}$, and is spatially discretized with a hexahedral mesh consisting of 770000 mesh elements, using the snappyHexMesh utility. The mesh, seen in figure 4 is refined in a $24 \mathrm{~m}$ high box around the water surface, and further refined in a box surrounding the buoy. A mesh resolution study was performed where the mesh was refined to a second, third and forth refinement level in the box closest to the buoy, seen in figure 5 (a), (b) and (c). 

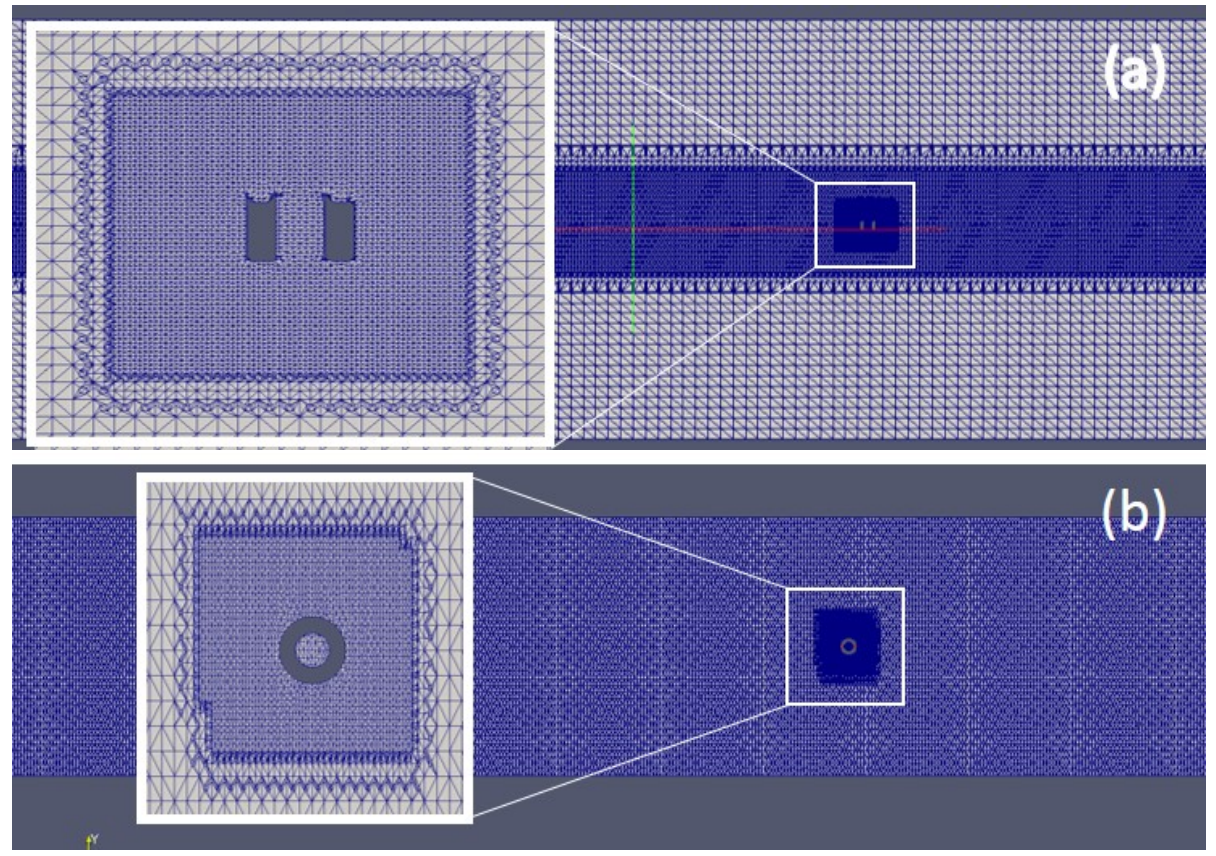

Figure 4: The mesh in the OpenFOAM model, side view (upper figure) and from above (lower).

In figure 5 (d), the resulting line force of the highest extreme wave is seen. The force is slightly higher for the finest mesh, and a fifth refinement level was studied as well. This resulted in the same line force, but the simulation was unsteady and a level four refinement was chosen. The waves in the model are generated and absorbed using the library waves2Foam, where relaxation zones are used to eliminate wave reflections from boundaries and internally [27. A relaxation zone of $150 \mathrm{~m}$ is placed at the inlet boundary, and a relaxation zone of $50 \mathrm{~m}$ is placed at the outlet boundary. Longer relaxation zones were tested initially, but since those short zones were seen to be sufficient, they were chosen. The PIMPLE algorithm is used, which combines the pressure-implicit split-operator (PISO) and the semi-implicit method for pressure-linked equations (SIMPLE). The forward Euler method is used to predict acceleration in the next time step.

In the OpenFOAM model, the WEC is simulated as a floating buoy moving 


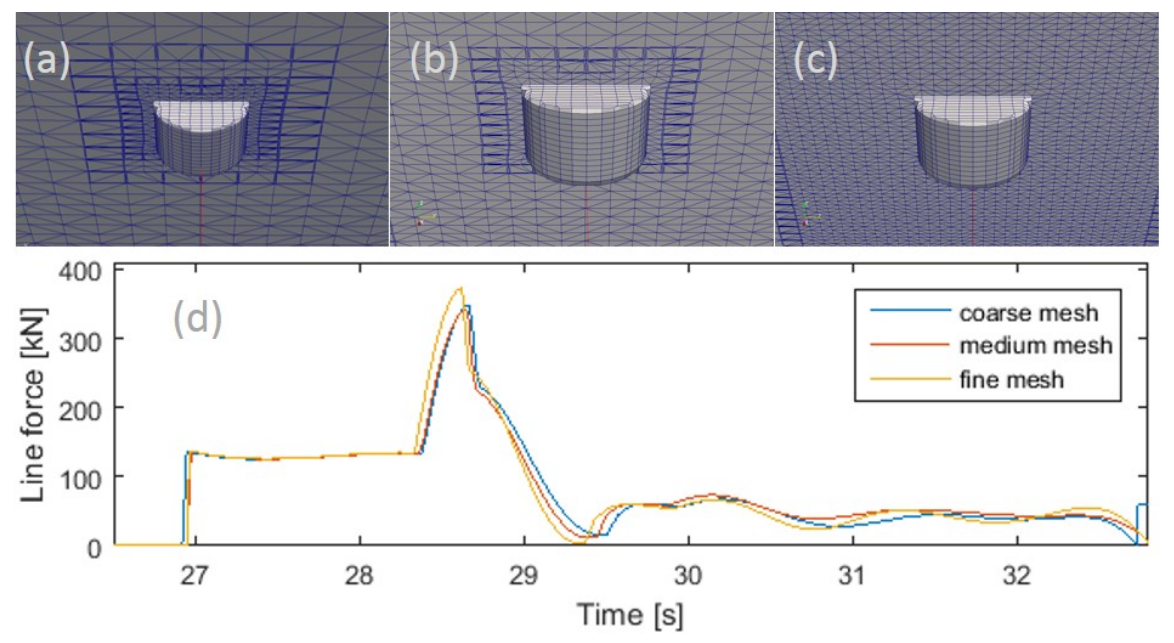

Figure 5: The mesh of the mesh resolution study in the OpenFOAM model and resulting force. (a) Coarse mesh. (b) Medium mesh. (c) Fine mesh. (d) Force in the connection line for the extreme wave.

in six degrees of freedom, restrained by a force directed along a line between the connection point at the buoy, $O_{b}$, and an anchor point on the seabed, $O_{t}$, The motion of the translator is not simulated separately, but the translator is simulated as a force in the connecting line, $F_{\text {line. }}$ The translator position is derived from the distance between $O_{b}$, and $O_{t}$;

$$
z_{t}=\left|O_{b}-O_{t}\right|-l_{\text {rest }}
$$

where $l_{\text {rest }}$ is the length of the connection line when the translator position is at equilibrium, $z_{t}=0$. The line force is then modelled as:

$$
F_{\text {line }}=m_{t} g-F_{\mathrm{PTO}}-F_{\kappa}-F_{\text {stop }}
$$

Since $F_{\mathrm{PTO}}$ is always directed in the opposite direction of the translator motion, a negative $F_{\text {line }}$ is possible, which would act as a lifting force on the buoy. $F_{\text {line }}$ is then set to zero, simulating a slack line.

\subsubsection{The ANSYS Fluent model}

The second model used in the paper solves for the hydrodynamical forces and the equations of motion in equation 1 using the commercial CFD package 

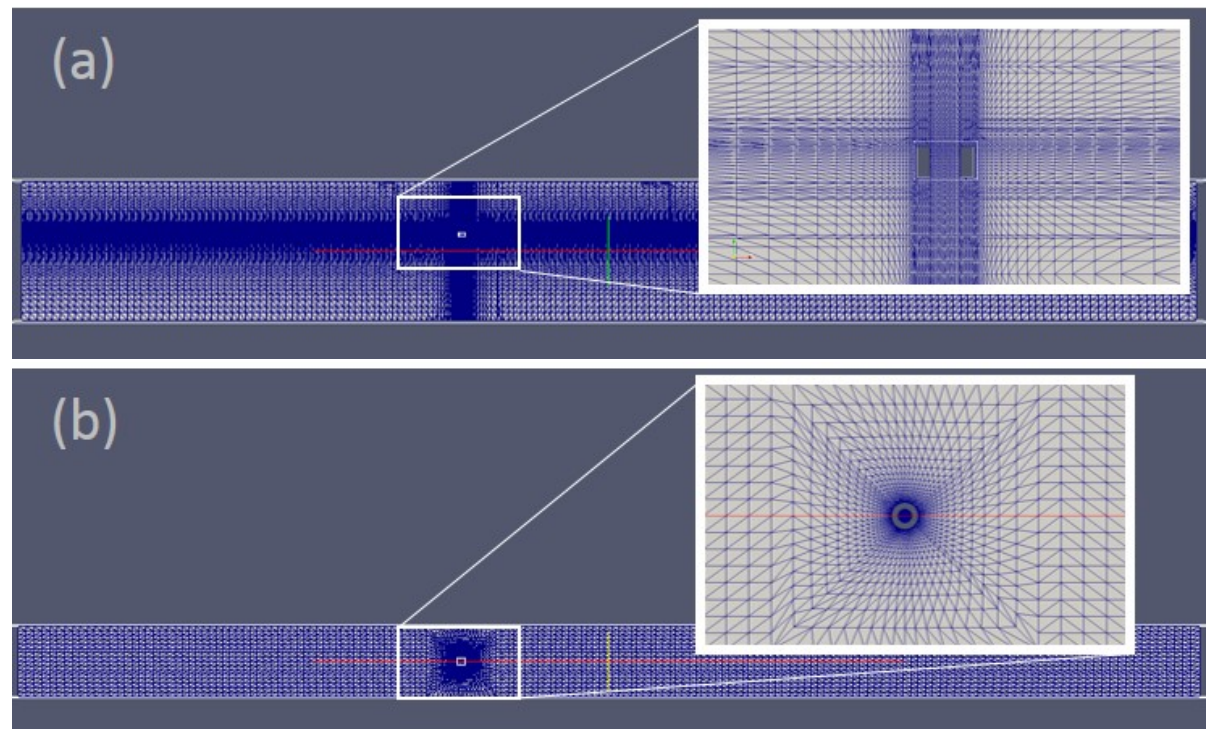

Figure 6: The mesh in the ANSYS model, side view (upper figure) and from above (lower).

Fluent 16.2. A numerical wave tank, $4 \lambda \times 40 \mathrm{~m} \times 80 \mathrm{~m}$, where $\lambda$ is the wavelength of the incident waves, is used to model the computational domain. The mesh is of structural type and seen in figure 6. A mesh resolution study was performed and is seen in figure 7 Three mesh resolutions were simulated and the buoy movement and force were compared. In figure 7 (d), the force in the connection line of the extreme wave is seen. The fine and the medium mesh showed good agreement, the same behaviour was seen for the buoy movement and the medium mesh was chosen. The numerical wave tank is discretized spatially using the widely used meshing tool Gambit, and the VOF is used to model the two fluids. The numerical wave tank with the CWM buoy consists of 320000 mesh elements, and 350000 mesh elements are used for the CYL buoy. The PISO pressurevelocity coupling scheme is used. In order to solve the second order differential equations in equation 1, the forward Euler method is used to predict acceleration in the next time step.

Waves are created at the inlet boundary by specifying water velocity distribution and wave height, while they are absorbed by damping water velocity 

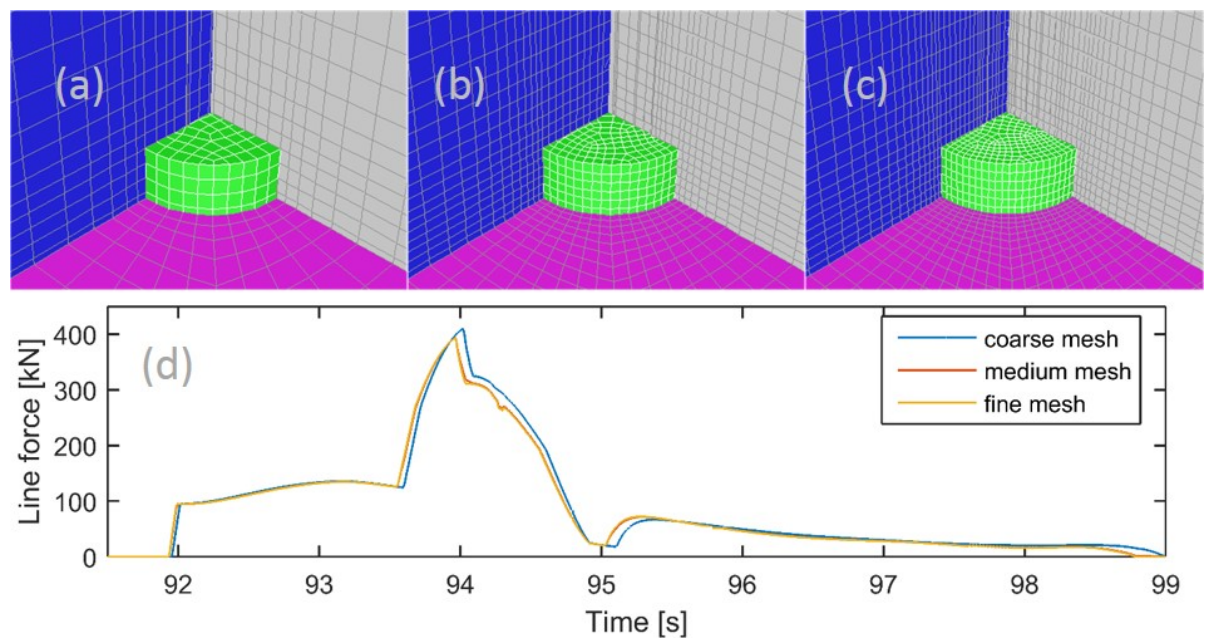

Figure 7: The mesh used in the mesh resolution study in the ANSYS model and resulting force. (a) Coarse mesh. (b) Medium mesh. (c) Fine mesh. (d) Force in the connection line for the extreme wave.

along the rear part of the wave tank over a length of one wavelength. The outlet and top of the wave tank are given a pressure-outlet boundary condition, and both sides of the wave tank use the symmetrical condition.

\subsection{Linear potential flow model}

For comparison with the two high fidelity CFD models, a simple and fast linear model has been developed, and is presented in this section. The linear model is a coupled time-domain model in Matlab, where the standard assumptions for linear potential flow theory are used: a non-compressible, homogeneous fluid density with negligible viscosity or vorticity is assumed. This reduces the governing equations to the Laplace equation $\Delta \phi=0$, where $\phi$ is the velocity potential and the fluid velocity is given by: $\bar{u}=\bar{\nabla} \phi$. Under the assumption of non-steep waves, the non-linear boundary condition at the free surface can be linearised and the first order approximation taken.

The wave-structure interaction can be described in terms of hydrodynamic forces obtained by the fluid pressure integrated over the wetted surface of the structure. The hydrodynamic forces can be divided into an excitation force re- 
sulting from the incident and scattered waves, and a radiation force originating from the waves radiated by the structure. The radiation force can be decomposed into two terms; one proportional to the acceleration (added mass) and one proportional to the velocity of the buoy (radiation damping). In general, the radiation force sums over all degrees of freedom and contains matrices where the nondiagonal terms represent the hydrodynamic coupling between oscillations in different degrees of freedom, e.g. surge and pitch motion. However, for an axisymmetric body such as a cylinder, most of the degrees of freedom are decoupled. In particular, this is true for the heave and surge motions, where only the diagonal terms of the radiation impedance are nonzero,

$$
\begin{aligned}
& F_{r, x}(t)=Z_{11}(t) * \dot{x}_{b}(t), \\
& F_{r, z}(t)=Z_{33}(t) * \dot{z}_{b}(t),
\end{aligned}
$$

where $Z(t)$ is the inverse Fourier transform of the modified impulse response function $Z(\omega)=i \omega m_{a}(\omega)+B(\omega)$ with the real part being the radiation damping $B(\omega)$ and the imaginary part being proportional to the added mass $m_{a}(\omega)$. Analogously, the excitation force can be written as a convolution with an impulse response to the incoming wave amplitudes,

$$
F_{e}(t)=f_{e}(t) * \eta(t)
$$

where $\eta(t)$ is the surface elevation of the incident waves.

For the linear model, we restrict the buoys motion to heave and surge only, so that the position of the buoy is parametrized by $\left(x_{b}(t), z_{b}(t)\right)$. Denote the constant distance between the free surface and the connection point of the translator by $l$. Introduce the parameter

$$
\varepsilon(t)=\frac{x_{b}(t)}{l+z_{b}(t)} \approx \frac{1}{l} x_{b}(t)
$$

describing the displacement in the $x$-direction relative to the vertical direction. Since $l$ is usually in the range $20-40 \mathrm{~m}$ and the displacements $x_{b}(t)$ and $z_{b}(t)$ are not larger than a few meters due to the limited stroke length of the translator, $\varepsilon(t)$ is a small parameter and we can make the assumption that all terms of the order $\varepsilon^{3}$ or smaller can be neglected. 
The connection line between the buoy and the translator can be modelled as a stiff spring. From the trigonometry of the problem, the line force on the buoy, in the $x$-direction, can be written as $F_{\text {line }, x}=\varepsilon F_{\text {line }, z}$ so that

$$
F_{\text {line }, x}=\varepsilon F_{\text {line }, z}=\frac{\varepsilon}{\sqrt{\left(1+\varepsilon^{2}\right)}} F_{\text {line }} \approx \varepsilon\left(1-\frac{1}{2} \varepsilon^{2}\right) F_{\text {line }}
$$

where $F_{\text {line }}$ is the magnitude of the line force. With these definitions, and using the trigonometric relations of the WEC model with a small parameter $\varepsilon$, the equations of motion become

$$
\begin{aligned}
& m_{b} \ddot{x}_{b}(t)=F_{e, x}(t)+F_{r, x}(t)-\varepsilon F_{\text {line }}(t)-C_{\text {drag }} \dot{x}_{b}\left|\dot{x}_{b}\right|, \\
& m_{b} \ddot{z}_{b}(t)=F_{e, z}(t)+F_{r, z}(t)-\left(1-\frac{1}{2} \varepsilon^{2}\right) F_{\text {line }}(t)+\rho g S\left(d-z_{b}(t)\right)-m_{b} g,
\end{aligned}
$$

where the excitation and radiation forces are computed as convolutions in the time-domain as in equations 10,11 , and the equation of motion for the translator is the same as given in equation (1). Under the assumption of a non-slack connection line between the buoy and the translator, the draft will be constant $d=\left(m_{b}+m_{t}\right) /(\rho S)$, where $S$ is the bottom surface area of the buoy. To improve accuracy due to the low radiation resistance for surge motion, a drag force term with constant coefficient, $C_{\text {drag }}$, has been added to the equation of motion in the $x$-direction according to the Morison equation [28]. The magnitude of the drag force coefficient has been investigated experimentally, and roughly lies in the range $C_{d} \in[0.10,1] \cdot \rho R d$ for slender cylinders in flows with high Reynolds number [29]. For the simulations presented here, a drag force of $C_{d}=0.625 \rho R d$ has been used, which corresponds to $C_{d}=1380 \mathrm{~kg} / \mathrm{m}$ for the CYL buoy and $C_{d}=2000 \mathrm{~kg} / \mathrm{m}$ for the CWM buoy. In addition to the PTO force in $F_{\text {line }}$, a low damping of $20 \mathrm{kN}$ is always applied in the linear model, which approximates the existing mechanical friction in the system.

Further, using the assumption of small parameter $\varepsilon$ in equation 12 , the 
vertical displacement of the translator is related to the position of the buoy as

$$
\begin{aligned}
z_{t}(t) & =\left(l+z_{b}(t)\right) \sqrt{1+\varepsilon(t)^{2}}-l \approx z_{b}(t)+\frac{1}{2} \varepsilon(t) x_{b}(t), \\
\dot{z}_{t}(t) & \approx \dot{z}_{b}(t)+\frac{1}{l} x_{b}(t) \dot{x}_{b}(t), \\
\ddot{z}_{t}(t) & \approx \ddot{z}_{b}(t)+\frac{1}{l}\left(x_{b}(t) \ddot{x}_{b}(t)+\dot{x}_{b}(t) \dot{x}_{b}(t)\right)
\end{aligned}
$$

as long as the buoy line is not slack. Inserting the expression for the line force from the equation of motion for the translator into the two equations for the buoy and neglecting terms smaller or equal to $\varepsilon^{3}$, the equations of motion become

$$
\begin{aligned}
m_{b} \ddot{x}_{b}(t)= & f_{e, x}(t) * \eta(t)-Z_{11}(t) * \dot{x}_{b}(t)-C_{\mathrm{drag}} \dot{x}_{b}\left|\dot{x}_{b}\right| \\
& -\varepsilon(t)\left[m_{t} \ddot{z}_{t}(t)+m_{t} g+\gamma \dot{z}_{t}(t)-F_{\text {stop }}(t)-F_{\kappa}(t)\right], \\
m_{b} \ddot{z}_{b}(t)= & f_{e, z}(t) * \eta(t)-Z_{33}(t) * \dot{z}_{b}(t)+\rho g S\left(d-z_{b}(t)\right)-m_{b} g \\
& -\left(1-\frac{1}{2} \varepsilon^{2}(t)\right)\left[m_{t} \ddot{z}_{t}(t)+m_{t} g+\gamma \dot{z}_{t}(t)-F_{\text {stop }}(t)-F_{\kappa}(t)\right] .
\end{aligned}
$$

Together with the expression for the translator position, velocity and acceleration (15), they describe the motion of the full system. By inserting the expressions for $z_{t}, \dot{z}_{t}$ and $\ddot{z}_{t}$ into 16 , a system of equations can be written in terms of the four parameters $x_{b}, z_{b}$ and their derivatives.

In the special case where the buoy only moves in heave, the horizontal equation of motion for the buoy in equation (14) vanishes, and $\varepsilon=0$. The line force on the translator in equation 13 equals the line force on the buoy, $F_{\text {line }, z}=F_{\text {line }}$ and the vertical displacement of the translator follows that of the buoy, $z_{t}(t) \approx z_{b}(t)$. The equations of motion for the translator and the buoy are connected by the spring force magnitude, and can be combined into a single equation which can be solved straight-forwardly in the frequency domain. This simplification is not used here, i.e. the buoy is free to move in surge and heave.

The coupled equations of motion in the time domain (16) can be reformulated in terms of ordinary differential equations of the first order and be solved numerically in Matlab using an ODE function. The hydrodynamic forces $Z_{11}$, $Z_{33}, f_{e, x}$ and $f_{e, z}$ have first been solved in the frequency domain using either 
the potential flow boundary element solver WAMIT or an analytical code, and then Fourier transformed to the time domain. At each time step, the Fourier transformed hydrodynamic forces are used in the convolutions (10)-11 for the velocities of the buoy and the incident waves, respectively. The excitation force in the surge direction is shifted $1.9 \mathrm{~s}$ in time to give the correct phase shift between the surge and heave responses. The incident waves in the simulations may be time-series of either regular or irregular waves. Despite the approximations, the model gives good results even for high amplitude waves, with a remarkably low computational cost, however only for the undamped case and for the CYL buoy, as will be discussed in section 3 .

\subsection{Physical wave tank experiment}

The models are compared with experimental wave tank data at 1:20 scale. In reference [23], the experiment is described in detail. An extreme wave event, embedded in regular waves, was produced in the COAST Laboratory Ocean Basin at Plymouth University, UK. The wave tank measures $35 \mathrm{~m} \times 15.5 \mathrm{~m}$ and the water depth was set to $2.5 \mathrm{~m}$. A 1:20 scale model of a WEC was built, with measurements corresponding to the parameters presented in table 1 . The position of the buoy was measured with an optical Qualisys system, consisting of five cameras outside the basin and four infrared markers attached to the buoy. The PTO was simplified as frictional damping, which was applied via adjustable Teflon blocks that were pressing on either side of the translator. It should be noted that when the frictional damping was applied, the motion of the translator was not smooth, but uneven. It is likely that this contributed to the somewhat scattered results in the measured motion and force data. At the top of the PTO unit, the translator motion was restricted by an endstop spring. The generator model was connected to the floating buoy using an $8 \mathrm{~m}$ long polymer line, which stretched $1 \%$ at a load of $60 \mathrm{~N}$ in 1:20 scale. The corresponding spring constant was calculated to $300 \mathrm{kN}$ at full scale, which is not high enough for the elasticity of the line to be neglectable. To account for this, an equivalent spring constant was calculated for the endstop spring, and 
when the endstop spring was fully compressed, the connection line functioned as a linear spring. The equivalent spring constant was calculated by combining the compressing endstop spring in series with the stretching line, which resulted in an equivalent spring constant of $489 \mathrm{kN} / \mathrm{m}$. However, when the translator did not hit the endstop spring, the line elasticity has been neglected.

\subsection{Incident waves}

The experiments presented in 23] studied a point-absorbing WEC in both regular, irregular and extreme waves. In this study, the same extreme wave embedded in a regular background has been used. The surface elevation incident on the buoy was measured by wave gauges in the experiments. This time-series of wave elevation has been used as an input to the excitation force convolution in the linear model. For the ANSYS and OpenFOAM models, frequency analysis

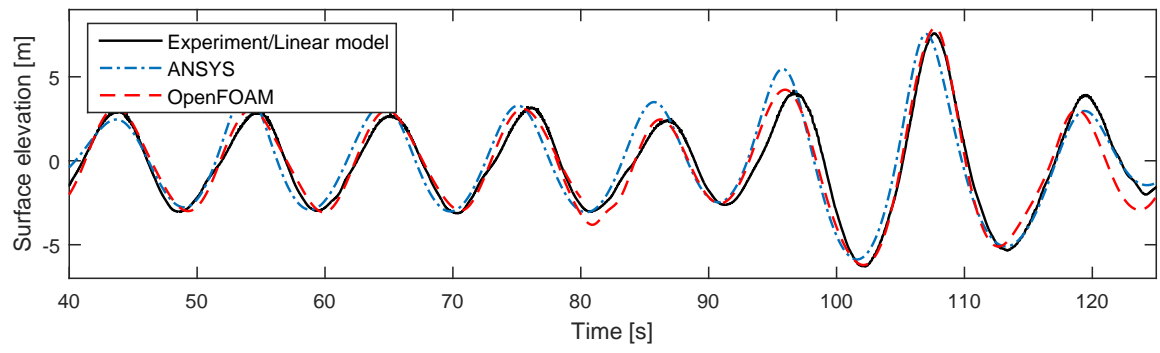

Figure 8: The surface elevation of the embedded focused wave.

of the embedded wave profile was performed to obtain the incident wave for the simulations. The four most significant wave components were superimposed to reproduce the experimental wave. In the ANSYS model, Heaviside functions were used to define the time period for different wave components to appear. For the OpenFOAM model, two separate simulations were run; first a monochrome wave, and then a separate simulation where the four wave components were superimposed. The resulting experimental and simulated waves at the position of the buoy are shown in figure 8 


\section{Results}

Simulating exact incident waves using CFD appears problematic. As can be seen from figure 8 , the wave height of the extreme wave is reproduced sat-

295 isfactorily, however, ANSYS Fluent slightly overestimates several of the wave peaks before the extreme event. OpenFOAM also overestimates the wave peaks, although to a smaller extent. In section 3.1 and 3.2 , the simulated buoy position and line force is compared to the measured experimental data in the time domain, where the different incident waves should be considered. In section 3.3 . 300 the peak forces have been plotted as a function of wave height, which makes it possible to compare force data from different incident waves.

\subsection{Buoy dynamics}

The modelled buoy displacement in heave and surge have been compared to the experimental data. In figure 9 the heave motion is presented for the CWM buoy in the case of no applied damping (a), and with frictional damping of 59 $\mathrm{kN}$ (b). It can be seen that the OpenFOAM model performs well in predicting the heave motion in both cases. The ANSYS Fluent model performs well for the undamped case, but underestimates the heave motion slightly for damped case. The linear model performs well in the undamped case, but overestimated the motion for the damped case.

In figure 10, the surge motion of the CWM buoy is presented for the cases of no applied damping (a), and $59 \mathrm{kN}$ of frictional damping (b). All models perform well for the low monochromatic waves, but only the ANSYS Fluent model is able to predict the surge motion during the extreme wave event. The OpenFOAM model underestimates the motion in the surge direction during the extreme event, while the linear model simulates a large motion in the negative surge direction. 


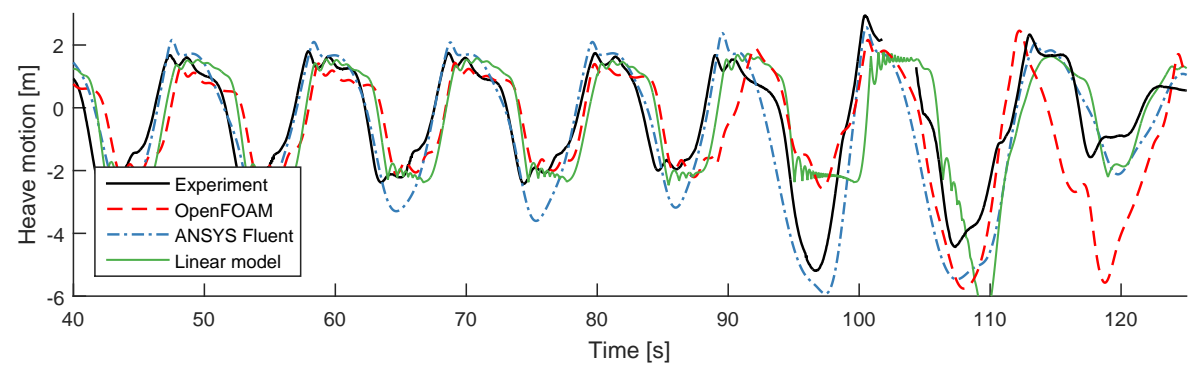

(a) No applied damping.

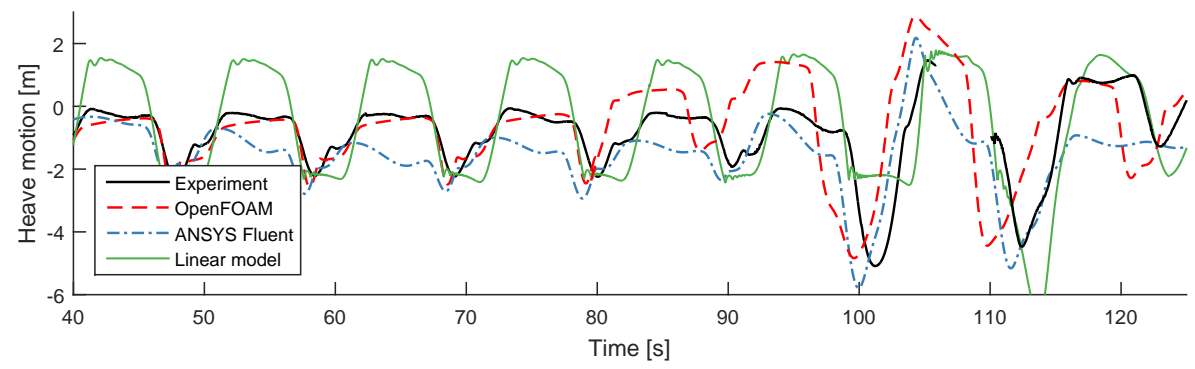

(b) $59 \mathrm{kN}$ frictional damping.

Figure 9: Experimental and simulated buoy position in heave motion. The CWM buoy was used. In the experiment, the Qualisys markers were submerged for a period of time during the extreme wave event, no experimental data is presented from this time period.

\subsection{Line force}

The simulated line force is compared to the experimental line force and presented in figure 11. Both the OpenFOAM model and the ANSYS Fluent model preform well for both the regular waves and the extreme wave event, although ANSYS Fluent slightly overestimates the force during the extreme wave for the CWM buoy. The linear model on the other hand, overestimated the force considerably throughout the time series.

\subsection{Peak forces as a function of wave height and applied frictional damping}

The incident wave in the CFD models had similar, but not the same, wave height as the incident wave in the wave tank and the linear model. To compare the peak forces of the different models, each peak force was plotted as a function

of the wave height of its corresponding wave peak. Figure 12 shows results for 


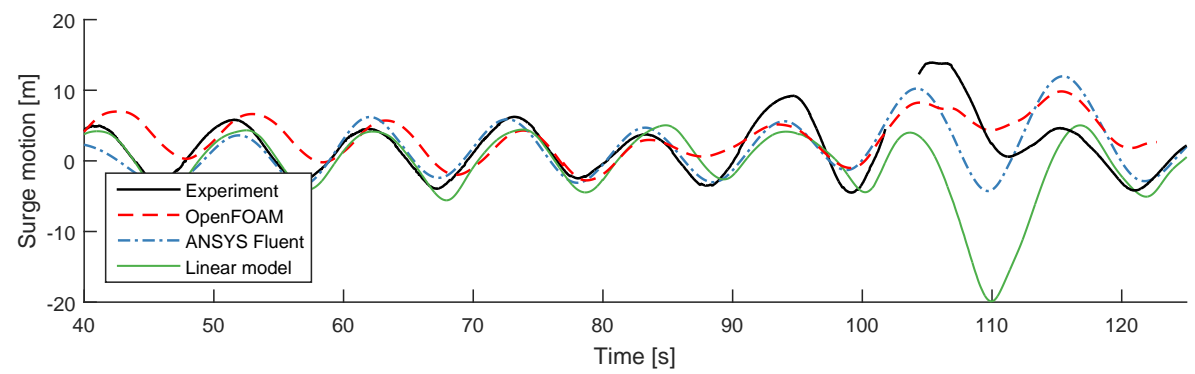

(a) No applied damping.

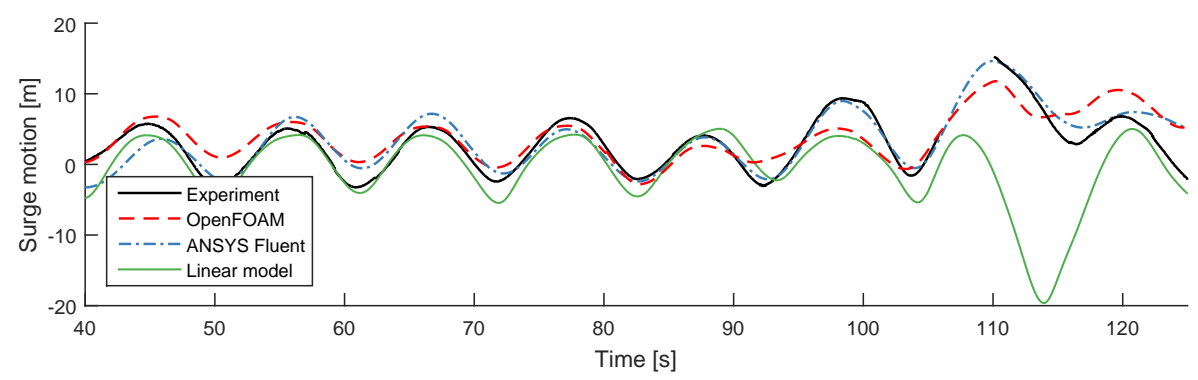

(b) $59 \mathrm{kN}$ frictional damping.

Figure 10: Experimental and simulated buoy position in surge motion. The CWM buoy was used. In the experiment, the Qualisys markers were submerged for a period during the extreme wave event, no experimental data is presented from this time period.

the CYL buoy, and figure 13 shows results for the CWM buoy. When studying the peak forces on the CYL buoy, in figure 12 , it can be seen that the force in both CFD models correspond well with the experimental force, for both regular waves and for the extreme wave event. The force peaks in the linear model correspond well with the experimental data when no damping is applied, although not for the extreme wave. The linear model overestimates the force peak of the extreme wave significantly, and when damping was applied, the linear model overestimated the force peaks of both the moderate waves and the extreme wave event.

For the CWM buoy, the force peaks are plotted in figure 13. Both OpenFOAM and ANSYS Fluent perform well in simulating the force peaks from both the regular waves and of the extreme wave event. For the extreme wave, the force peak is slightly underestimated, which possibly is an effect of how the 


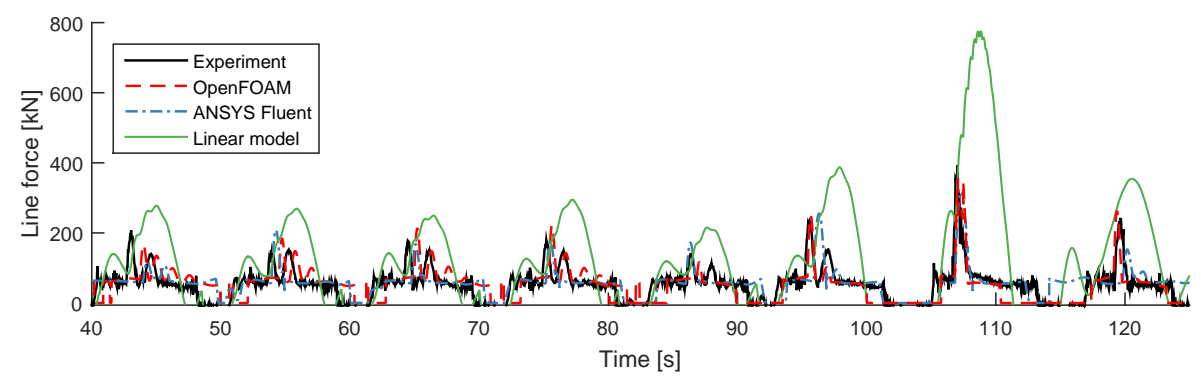

(a) No damping applied.

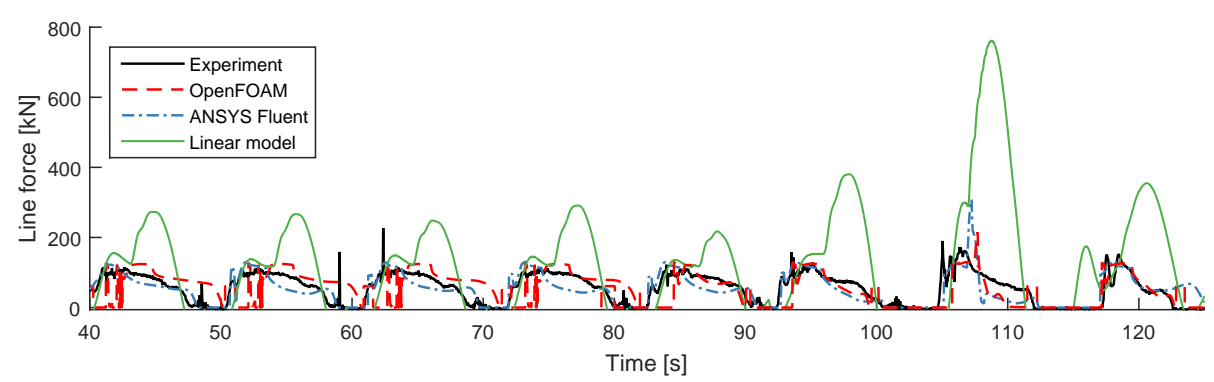

(b) $59 \mathrm{kN}$ frictional damping.

Figure 11: Experimental and simulated line force. The CWM buoy was used.

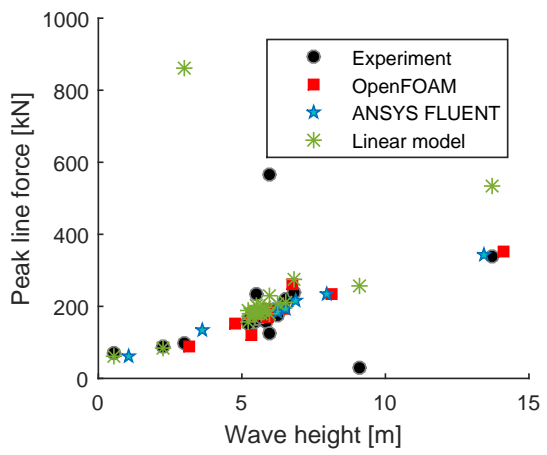

(a) No damping applied.

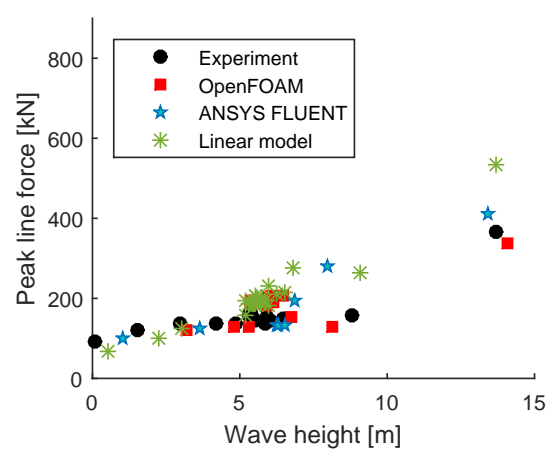

(b) $59 \mathrm{kN}$ frictional damping.

Figure 12: Peak forces for the WEC with the CYL buoy. Each peak force is plotted as a function of the wave height of its corresponding wave peak.

PTO-system was simulated. The magnitude of the force peak depends directly on the spring constant of the connection line when the endstop spring is fully compressed, and it is possible that the spring constant in the experiment was 


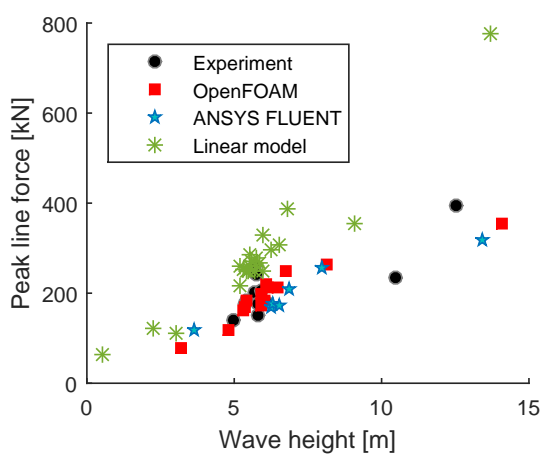

(a) No damping applied.

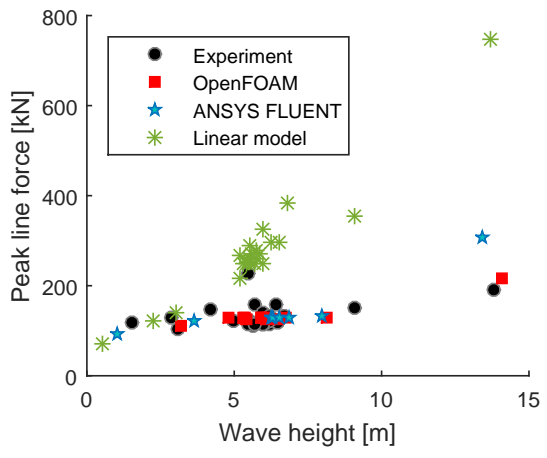

(c) $59 \mathrm{kN}$ frictional damping.

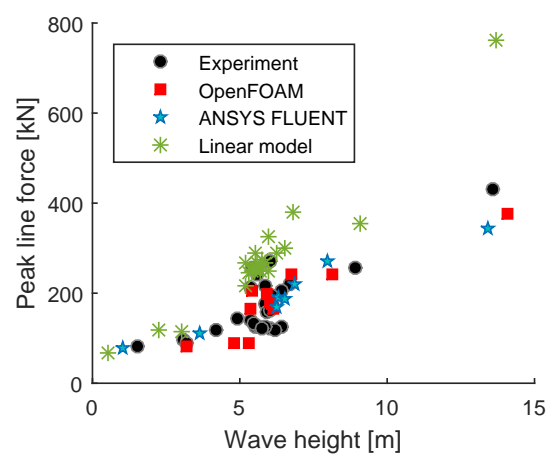

(b) $18 \mathrm{kN}$ frictional damping.

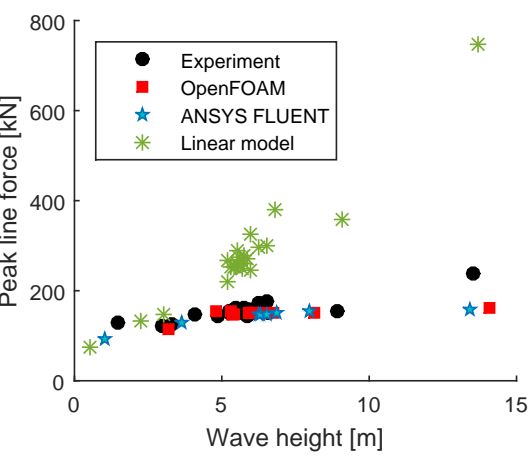

(d) $83 \mathrm{kN}$ frictional damping.

Figure 13: Peak forces for the WEC with the CWM buoy. Each peak force is plotted as a function of the wave height of its corresponding wave peak.

increased in the extreme wave; since it was already stretched due to the high load it might have exceeded its linear region.

As was studied in [23, the magnitude of the peak forces decreases with an increased damping. When no damping was applied and in the case of $18 \mathrm{kN}$ damping, the force increases with increased wave height. For the two higher damping cases the peak forces reach a limit and level out when the buoy is overtopped by the waves. Both CFD models capture this behaviour from the physical experiment, whereas the peak forces of the linear model do not level out for the higher damping cases. 


\subsection{Model performance and computational cost}

As is seen in figure 12 and figure 13 , the model performance differs between the different simulations. The error of the simulated peak line force has been calculated for each wave peak (i) as:

$$
\text { Error(i) }=\frac{\left|F_{\text {peak, exp }}^{(i)}-F_{\text {peak, sim }}^{(i)}\right|}{F_{\text {peak, exp }}^{(i)}} .
$$

The errors have been compared to simulation time in figure 14 , where an average has been calculated for the moderate waves, and the extreme wave peaks are presented separately.
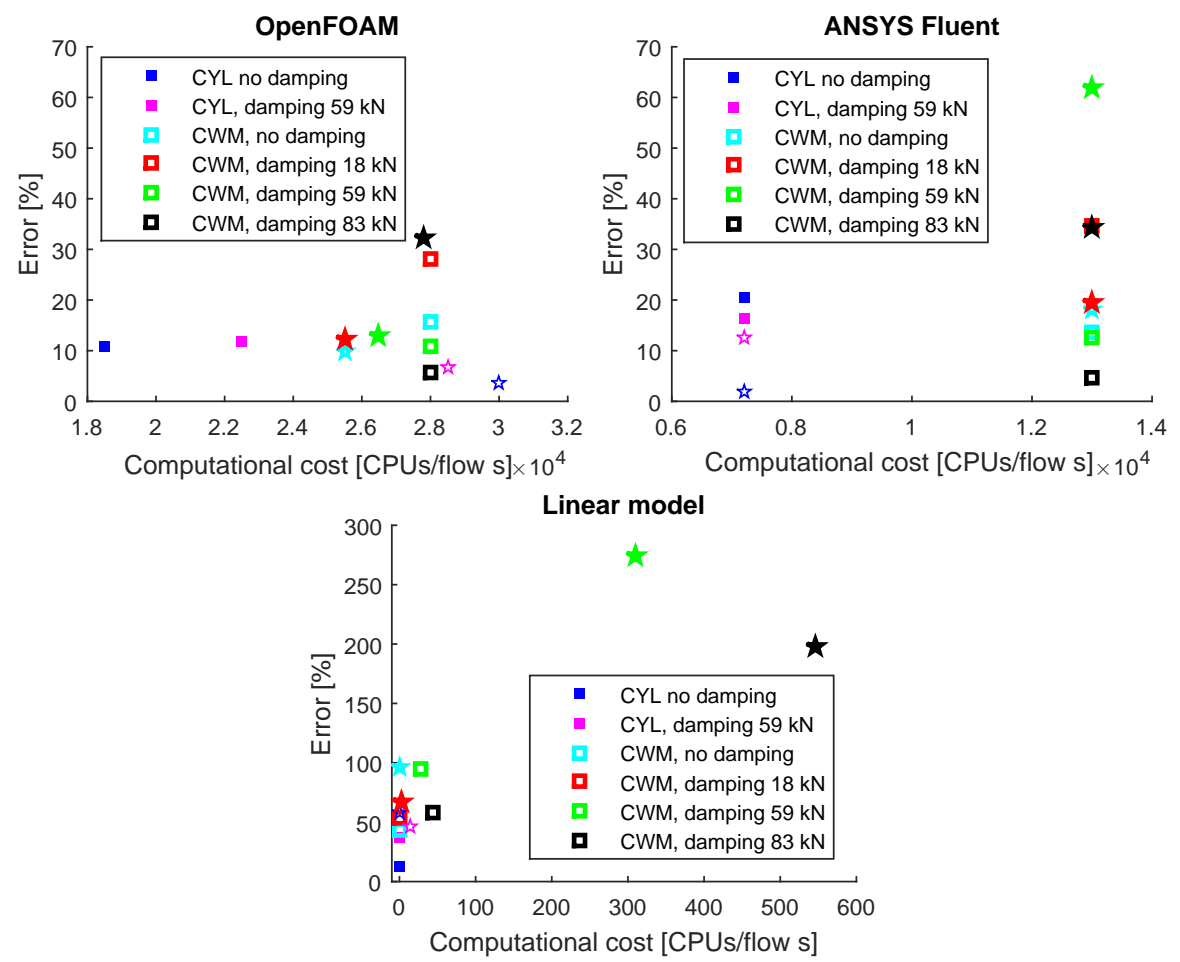

Figure 14: The error of the simulated peak force is shown as a function of computational cost. The average error of the moderate waves are represented by squares, filled for CYL and unfilled for CWM, while the extreme wave are represented by filled and unfilled stars. Please note that the $\mathrm{x}$-axis is truncated for visibility. 
It can be seen that although the computational cost is very low for the linear simulation, the error is not acceptable for cases other than for the CYL buoy without applied damping. It can also be seen that although the computational cost is lower for ANSYS Fluent than for OpenFOAM, the choice of OpenFOAM can be justified by a higher accuracy. The average simulation errors for each model were $13 \%$ for OpenFOAM, $21 \%$ for ANSYS Fluent and $87 \%$ for the linear model.

\subsection{Mesh comparison}

The mesh used in ANSYS Fluent is built using the widely used meshing tool GAMBIT, while snappyHexMesh is used for the OpenFOAM mesh. To provide a more qualitative comparison between the CFD models, the Gambit mesh is imported in OpenFOAM to study the mesh dependency of the results, and is compared with the OpenFOAM model with SnappyHexMesh and with the ANSYS Fluent model. Figures 15 and 16 show the results from the CWM buoy with no applied damping and $59 \mathrm{kN}$ of damping. The surface elevation of the incident wave depends on the mesh, which results in differences in both buoy motion and line force. When the Gambit mesh was used with the OpenFOAM model, the simulation crashed after the highest extreme wave, due to highly sheared mesh elements during the large surge motion.

\section{Discussion}

380

The linear model predicts the buoy motion well, in both heave and surge, when no PTO damping is applied, and for low and moderate waves. However, the performance of the model was dependent on both buoy geometry and PTO damping. When damping was applied, the model overestimated the heave motion, while the surge motion still showed a good agreement with the experiment.

385 The force in the connection line showed acceptable agreement with the physical experiment for the CYL buoy in moderate waves, but was overestimated for the extreme wave event and for the CWM buoy. It is concluded that the performance of the linear model is highly dependent on both buoy geometry and the 

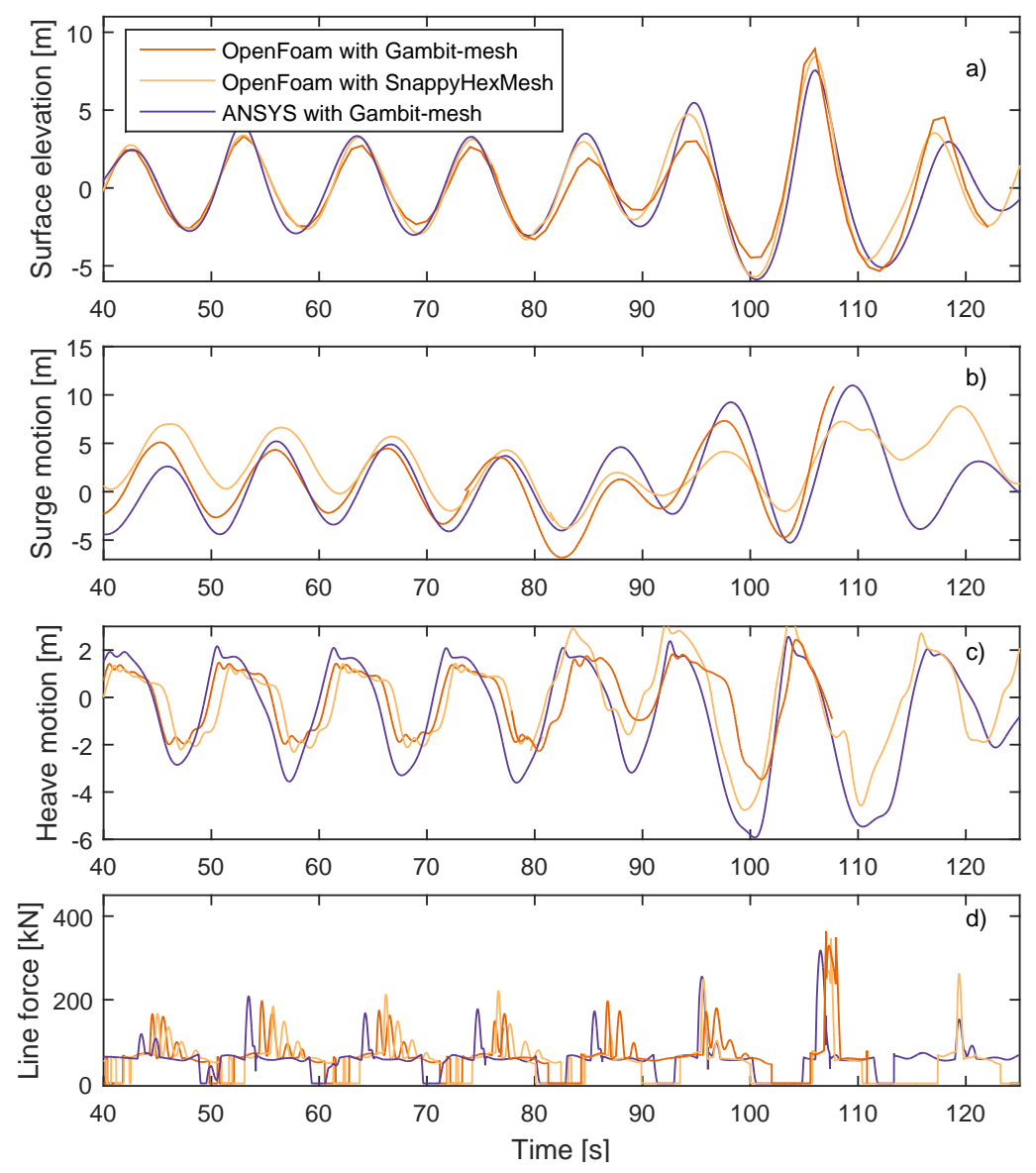

Figure 15: Mesh comparison for the CWM buoy with no damping applied. 

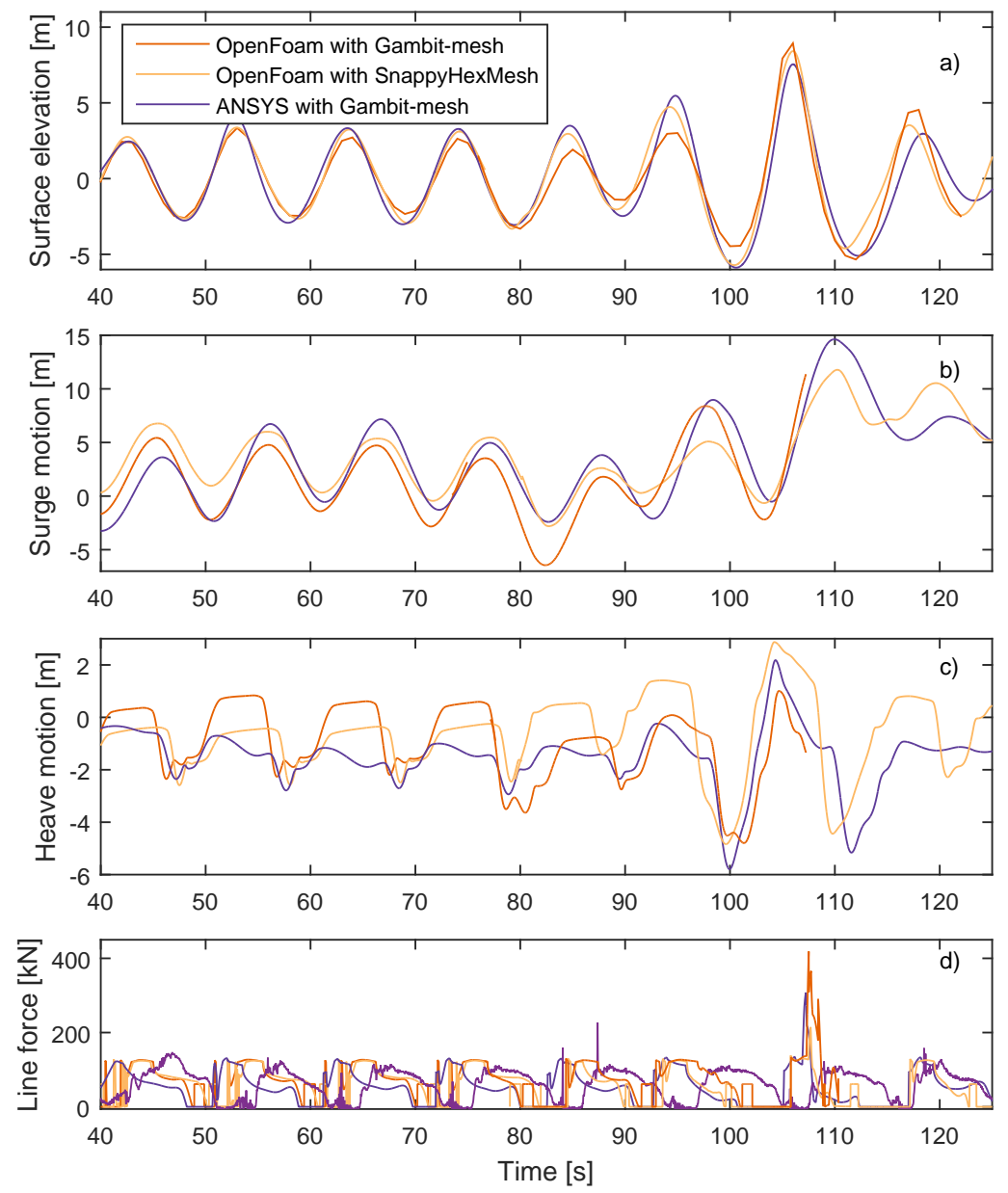

Figure 16: Mesh comparison for the CWM buoy with applied frictional damping of $59 \mathrm{kN}$. 
level of applied damping. The line force of the extreme wave was expected to comparison of the models. However, the meshes are built in meshing tools that are widely used for each simulation software, and since mesh resolution studies are made for each mesh, we find the comparison interesting as this is how the 
the two meshes using the OpenFOAM model, the largest difference was found in implementing the same incident wave, which resulted in differences in motion and force. This highlights that one of the difficulties in using CFD models is implementing predefined incident waves.

425

It can be seen that the experimental peak forces show a somewhat scattered result when plotted as a function of wave height. This is expected, since the peak force does not only depend on the incident wave height, but also on the previous wave, since the previous wave will affect the present velocity and acceleration of both the buoy and the translator. It will also depend on the wave frequency and of course on the behavior of the mooring, in this case the PTOsystem. The scattering is smaller for the CYL buoy than for the CWM buoy, and it decreases with increased PTO-damping. A scattering behavior is seen in all the simulation models but to a lower extent than for the physical experiment. to increasing the calculated error of the simulation models; in this paper each physical peak force is compared to a corresponding simulated peak force. In figure 13, it can be seen that the trends in the physical experiment is captured to a high extent by both the CFD models, but the scatted results of the physical experiments increases the error. It is seen that if trend lines would have been used for the error estimations, the error would be smaller for the CFD models.

As was studied in reference 23], the magnitude of the peak forces decreases with an increased damping, which is a consequence of a decreased translator speed when the endstop spring was impacted 30. However, comparing no added damping with low added damping, figure 13 (a) and (b), the added damping does not seem to decrease the peak forces to the same extent as when higher damping was added. One possible explanation can be that the low applied frictional damping is acting as latching; it initially holds the translator and buoy 450 in position and increase the phase shift between the buoy and the wave, which 
increases the translator speed and the force in the endstop hit. Studying the two highest damping cases, figure 13 (c) and (d), it is seen that the force first increases with wave height and then levels out. In reference [31, this behaviour was seen when the buoy was overtopped by waves, but only when the applied damping was high enough to keep the translator from hitting the endstop spring. If the endstop spring was hit, higher waves resulted in higher peak forces, regardless if overtopping occurred or not. However, the highest damping studied in 31. was only $30 \mathrm{kNs} / \mathrm{m}$, and a higher damping might decrease the peak forces even when the endstop spring is hit, which was seen in this paper when $59 \mathrm{kN}$ and $83 \mathrm{kN}$ of applied damping.

\section{Conclusions}

The influence of frictional damping on the peak forces of a WEC has been studied using two CFD models, and the results have been confirmed with physical wave tank data. One linear model have also been presented and compared with the CFD models and the physical experiment. It was seen that the peak forces in high and extreme waves were decreased by an increased PTO damping. One of the challenges in the CFD simulations was to implement the exact incident wave as in the physical experiments, which contributed to the seen differences between the CFD results and the physical wave tank result. When the peak forces were plotted as a function of wave height, good agreement was found between both CFD models and the physical wave tank data. Both CFD models were considered verified and can be used for studying the motion and force of WECs with good experimental agreement, as long as the behavior of the PTO system can be correctly described.

It was also seen that the model performance was dependent on both applied load and buoy geometry. This was seen especially for the linear model, where good agreement with the physical experiment was found when no damping was applied and for the simplest buoy geometry, but the model performance was poor for damped cases and for the buoy with a moonpool. 
Acknowledgment

The research was supported by Centre for Natural Disaster Science (CNDS) in Sweden, the Chinese Scholarship Council (CSC), the Swedish Research Council (VR) grant 2015-04657, Bengt Ingeströms scholarship fund and the Wallenius fund. The computations were performed on resources provided by the Swedish 485 National Infrastructure for Computing (SNIC) at UPPMAX.

\section{References}

[1] S. Salter, Wave power, Nature 249 (1974) 720-724.

[2] K. Budal, J. Falnes, A resonant point absorber of ocean-wave power, Nature 256 (1975) 478-479.

[3] C. Mei, Power extraction from water waves, Journal of Ship Research 20 (1976) 63-66.

[4] D. Evans, A theory for wave-power absorption by oscillating bodies, Journal of Fluid Mechanics 77 (1976) 1-25.

[5] J. Falnes, A review of wave-energy extraction, Marine Structures 20 (2007) $185-201$.

[6] A. Falcao, Wave energy utilization: a review of the technologies, Renewable and Sustainable Energy Reviews 14 (2010) 899-918.

[7] D. Evans, Power from water waves, Ann. Rev. Fluid Mechanics 13 (1981) $157-187$.

[8] E. Jefferys, Device characterisation, Power from Sea Waves (1980) 413-438.

[9] T. B. G. Payne, J. Taylor, P. Parkin, Assessment of boundary element method for modelling a free-floating sloped wave energy device. part 2 : Experimental verification, Ocean Engineering 35 (2008) 342-357. 
[10] L. Sjökvist, R. Krishna, V. Castellucci, A. Hagnestål, M. Rahm, M. Leijon, On the optimization of point absorber buoys, Journal of Marine Science and Engineering 2(2) (2014) 477-492.

[11] H. Wolgamot, C. Fitzgerald, Nonlinear hydrodynamic and real fluid effects on wave energy converters, In Proc IMechE, Part A: J Power and Energy (2015).

[12] C. Eskilsson, A. Bosi, M. Ricchiuto, Wave induced motion of pointabsorbers: a hierarchial investigation of hydrodynamic models, In proceedings of the 11th European Wave and Tidal Energy Conference Series EWTEC, Nantes, France (2015).

[13] J. Davidson, S. Giorgi, J. Ringwood, Linear parametric models for wave enrgy converters identified from numerical wave tank experiments, Ocean Engineering 103 (2015) 31-39.

[14] J. Davidson, S. Giorgi, J. Ringwood, Identification of wave energy device models from numerical wave tank data - part 1: Numerical wave tank identification tests, IEEE Transactions on Sustainable Energy 7 (2016) 1012 $-1019$.

[15] P. Schmitt, B. Elsaesser, On the uso of openfoam to model oscillating wave surge converters, Ocean Engineering 108 (2015) 98-104.

[16] Y. Yu, Y. Li, Reynolds-averaged Navier-Stokes simulation of the heave performance of a two-body floating-point absorber wave energy system, Computers \& Fluids 73 (2013) 104-114.

[17] E. Ransley, Survivability of wave energy converter and mooring coupled system using CFD, Ph.D. thesis, Plymouth University (2015).

[18] E. Ransley, D. Greaves, A. Raby, D. Simmonds, M. Hann, Survivability of wave energy converters using CFD, Renewable Energy (2017). 
[19] E. Ransley, D. Greaves, A. Raby, D. Simmonds, M. Jakobsen, M. Kramer, RANS-VOF modelling of the wavestar point absorber, Renewable Energy (2017).

[20] M. Leijon, C. Bodström, O. Danielsson, S. Gustafsson, K. Haikonen, O. Langhamer, E. Strömstedt, M. Stalberg, J. Sundberg, O. Svensson, S. Tyrberg, R. Waters, Wave energy from the north sea: Experiences from the lysekil research site, Surv Geophysics 29 (2008) 221-240.

[21] A. Savin, O. Svensson, E. Strömstedt, C. Boström, M. Leijon, Determining the service life of a steel wire under a working load in the wave energy converter, Proceedings of the ASME 28th International Conference on Ocean, Offshore and Arctic Engineering, OMAE2009 Honolulu, Hawaii.

[22] M. L. O. Svensson, Peak force measurements on a cylindrical buoy with limited elastic mooring, IEEE Journal of Oceanic Engineering 39(2) (2014) $398-403$.

[23] M. Göteman, J. Engström, M. Eriksson, M. Hann, E. Ransley, D. Greaves, M. Leijon, Wave loads on a point-absorbing wave energy device in extreme waves, Journal of Ocean and Wind Energy 2 (2015) 176-181.

[24] W. Chen, I. Dolguntseva, A. Savin, Y. Zhang, W. Li, O. Svensson, M. Leijon, Numerical modelling of a point-absorbing wave energy converter in irregular and extreme waves, Applied Ocean Research 63 (2017) 90-105.

[25] A. Day, A. Babarit, A. Fontaine, Y. He, M. Kraskowski, M. Murai, I. Penesis, F. Salvatore, H. Shin, Hydrodynamic modeling of marine renewable energy devices: A state of the art review, Ocean Engineering 108 (2015) $46-69$.

[26] R. Waters, J. Engström, J. Isberg, M. Leijon, Wave climate off the swedish west coast, Renewable Energy 34 (2009) 1600-1606. 
[27] J. F. Niels G. Jacobsen, David R. Fuhrman, A wave generation toolbox for the open-source CFD library: Openfoam, International Journal for Numerical Methods in Fluids 70 (2012) 1073-1088.

[28] J. Morison, J. Johnson, S. Schaaf, et al., The force exerted by surface waves on piles, Journal of Petroleum Technology 2 (05) (1950) 149-154.

[29] T. Sarpkaya, M. Isaacson, J. Wehausen, Mechanics of wave forces on offshore structures, Journal of Applied Mechanics 49 (1982) 466.

[30] L. Ulvgård, L. Sjökvist, M. Göteman, M. Leijon, Line force and damping at full and partial stator overlap in a linear generator for wave power, Journal of Marine Science and Engineering 4.

[31] L. Sjökvist, M. Göteman, The effect of overtopping waves on peak forces on a point absorbing WEC, In proceedings of Asian Wave and Tidal Energy Conference Series AWTEC, Singapore (2016). 